\title{
О КОЛИЧЕСТВЕ ПАРАМЕТРОВ МНОГОМЕРНОЙ ДИНАМИЧЕСКОЙ СИСТЕМЫ
}

\section{Введение}

Рассмотрим линейную многомерную систему

$$
\begin{gathered}
x(t+1)=A x(t)+B u(t), \\
y(t)=C x(t),
\end{gathered}
$$

где $u, x$ и $y$ - векторы входа, состояния и выхода размерности $m, n$ и $p$ соответственно, а $A, B$ и $C$ - матрицы подходящей размерности.

Сложность идентификации динамического объекта, а также синтеза системы управления во многом зависит от количества неизвестных параметров в их описаниях. Поэтому много внимания уделяется изучению канонических форм описания (1), позволяющих заранее фиксировать некоторые элементы матриц $A, B$ и $C$. Разнообразность канонических форм обусловлена неединственностью описания (1). А именно, изменение базиса пространства состояния не влияет на соотношение между входом и выходом, т. е. матрицы $A, B$ и $C$ в описании (1) можно заменить матрицами $\widetilde{A}, \widetilde{B}$ и $\widetilde{C}$, найденными по формулам

$$
\begin{aligned}
\widetilde{A} & =T A T^{-1}, \\
\widetilde{B} & =T B, \\
\widetilde{C} & =C T^{-1},
\end{aligned}
$$

где $T$ - любая невырожденная матрица порядка $n \times n$.

В общем случае матрицы $A, B$ и $C$ содержат $n(n+m+p)$ неизвестных параметров. Ввиду инвариантности отображения вход-выход относительно линейного преобразования $T$ на пространстве состояния $n^{2}$ параметров тройки $(A, B, C)$ могут быть приведены к ранее выбранным значениям (0 и 1) [ $\left.{ }^{1}\right]$. Значит, если удается определить расположение $n^{2}$ фиксированных параметров в описании (1), тогда число неизвестных параметров будет удовлетворять неравенству

$$
N \leqslant n(m+p) .
$$

Фиксирование значений и расположения некоторого числа параметров в описании (1) приводит его к определенной канонической форме.

В данной статье фиксируются элементы матриц $A$ и $C$ при произ- 
вольной матрице $B$. Дуальным подходом можно добиться аналогичных соотношений для фиксирования элементов матриц $A$ и $B$ при произвольной матрице $C$. Изучается каноническая форма Луэнбергера, как наиболее распространенная при исследовании линейных систем. Выводятся общие формулы для определения числа неизвестных параметров, а также фиксируется их расположение по информации, необходимой для установления структуры канонической формы. Выясняются наиболее и наименее благоприятные структуры канонических форм и соответствующие им минимальные и максимальные числа неизвестных параметров.

\section{Каноническая форма Луэнбергера}

Пусть система (1) минимально наблюдаема, т. е. все $p$ выходов необходимы для наблюдения системы $\left[{ }^{2}\right]$. Тогда система (1) приводится к канонической форме Луэнбергера $\left[{ }^{3}\right]$

$$
\begin{aligned}
& \widetilde{A}=\left[A_{i j}\right], \quad i, j=1, \ldots, p, \\
& \underset{\left(n_{i} \times n_{i}\right)}{A_{i i}}=\left[\begin{array}{cccc}
0 & 1 & 0 \ldots & 0 \\
0 & 0 & 1 \ldots & . \\
\cdot & . & & . \\
\cdot & \cdot & & \cdot \\
a_{i i 1} & \dot{a}_{i i 2} & \ldots & \dot{a}_{i i n_{i}}
\end{array}\right] \text {, }
\end{aligned}
$$

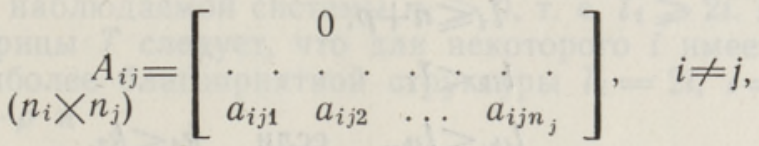

$$
\begin{aligned}
& \widetilde{C}=\left[\begin{array}{l}
e_{1}^{T} \\
e_{n_{1}+1}^{T} \\
\vdots \\
\vdots \\
e_{n_{1}+\ldots+n_{p-1}+1}^{T}
\end{array}\right] \\
& e_{i}^{T}=\left[\begin{array}{llllll}
0 \ldots & \ldots & 1 & 0 & \ldots & 0
\end{array}\right] \text {, }
\end{aligned}
$$

преобразованием

$$
T=\left[\begin{array}{c}
T_{1} \\
T_{2} \\
\cdot \\
\dot{T}_{p}
\end{array}\right], \quad T_{i}=\left[\begin{array}{c}
c_{i}^{T} \\
c_{i}^{T} A \\
\vdots \\
\vdots \\
c_{i}^{T} A^{n_{t}-1}
\end{array}\right]
$$

Структура канонической формы (4), (5) определяется индексами $n_{i}$, $i=1, \ldots, p$, причем

$$
\sum_{i=1}^{p} n_{i}=n
$$


Структурные индексы $n_{i}$, которые находятся путем подходящего выбора матрицы $T$ из соотношения (6) [ $\left.{ }^{3}\right]$, позволяют фиксировать значения и расположение $n^{2}$ параметров в канонической форме (4), (5), т. е. они позволяют установить верхнюю границу неравенства (3).

Представляет интерес выяснить возможности дальнейшего уменьшения числа неизвестных параметров априорным фиксированием некоторых элементов $a_{i j k}$. Оказывается, что конкретные значения параметров $a_{i j k}$, а также структурных индексов $n_{i}$ зависят от последовательности выбора строк матрицы $T$ из матрицы наблюдаемости

$$
\mathfrak{D}^{T}=\left[C^{T}, A^{T} C^{T}, \ldots,\left(A^{n-1}\right) C^{T}\right] .
$$

Целесообразно выделить два этапа в процедуре выбора строк матрицы $T$ :

1) некоторая строка $c_{i}^{T} A^{k}$ матрицы $D$ становится кандидатом в матрицу $T$, если строки $c_{i}^{T}, c_{i}^{T} A, \ldots, c_{i}^{T} A^{k-1}$ уже вошли в состав матрицы $T$,

2) кандидат включается в матрицу $T$, если он не зависит линейно от ранее выбранных строк матрицы $T$.

Пронумеруем все кандидаты в порядке их выдвижения. Пусть $l_{i k}$ и $\bar{l}_{i}, i=1, \ldots, p, k=1, \ldots, n_{i},-$ порядковые номера включенных и невключенного кандидатов, связанных с $i$-м выходом. Тогда в силу 1) и 2) выполняются следующие неравенства:

$$
\begin{aligned}
\bar{l}_{i} & \leqslant n+p, \\
l_{i k} & <\bar{l}_{i}, \\
l_{i k_{1}} & <l_{i k_{2}}, \text { если } \quad k_{1}<k_{2} .
\end{aligned}
$$

Из процедуры выбора матрицы $T$ следует, что строка $c_{i}^{T} A^{n_{t,}, \text { являю- }}$ щаяся $\bar{l}_{i \text {-м }}$ кандидатом, может быть представлена суммой

$$
c_{i}^{T} A^{n_{t}}=\sum_{s=1}^{p} \sum_{r=0}^{n_{s-1}} \alpha_{i s r} c_{s}^{T} A^{r},
$$

причем

$$
\alpha_{i s r}=0, \quad \text { если } \quad l_{s r}>\bar{l}_{i},
$$

где $l_{s r}$ - порядковый номер кандидата $c_{s}^{T} A^{r-1}$. Обозначим

$$
\begin{aligned}
T^{-1} & =\left[D_{1}, \ldots, D_{p}\right], \\
D_{j} & =\left[d_{j 1}, \ldots, d_{j n_{j}}\right] .
\end{aligned}
$$

Тогда из уравнений $(2),(4),(6)$ и (12) получим

$$
a_{i j k}=c_{i}^{T} A^{n_{\iota}} d_{j k},
$$

а с учетом (10) соответственно

Так как

$$
a_{i j k}=\sum_{s=1}^{p} \sum_{r=0}^{n_{s-1}} \alpha_{i s r} c_{s}^{T} A^{r} d_{j k} .
$$




$$
c_{s}^{T} A^{r} d_{j k}=\left\{\begin{array}{lll}
1, & \text { если } s=j, & r=k-1, \\
0 & \text { в противном случае }
\end{array}\right.
$$

то из уравнения (13) следует

$$
a_{i j k}=\alpha_{i j(k-1)}
$$

Равенства (11) и (14) фиксируют дополнительные нули в матрице $\widetilde{A}$

$$
a_{i j k}=0, \text { если } l_{j k}>\bar{l}_{i}, \quad j=1, \ldots, p, k=1, \ldots, n_{j} .
$$

Согласно (15) число неизвестных параметров в матрице $\tilde{A}$ сокращается до

$$
\sum_{i=1}^{p}\left(\bar{l}_{i}-i\right)
$$

а в канонической форме Луэнбергера до

$$
N=n m+\sum_{i=1}^{p} \bar{l}_{i}-p(p+1) / 2 .
$$

Из формулы (16) видно, что для минимизации числа $N$ следует выбирать строки матрицы $T$ в такой последовательности, чтобы $\sum_{i=1}^{p} \bar{l}_{i}=\min$.

Для минимально наблюдаемой системы $n_{i}>0$, т. е. $\bar{l}_{i} \geqslant 2 i$. Из процедуры выбора матрицы $T$ следует, что для некоторого $i$ имеет место $\bar{l}_{i}=n+p$. Для наиболее благоприятной структуры $\bar{l}_{i}=2 i, i=1, \ldots$ $\ldots, p-1, \bar{l}_{p}=n+p$ и

$$
N_{\min }=n(m+1)+p(p-1) / 2 .
$$

Для наихудшей структуры из неравенства (9) и формулы (16) получим $\bar{l}_{i}=n f i, i=1, \ldots, p$ и

$$
N_{\max }=n(m+p) \text {. }
$$

\section{Разновидности канонической формы Луэнбергера}

Рассмотрим две разновидности канонической формы Луэнбергера, которые наиболее удобны для составления матрицы $T[2,4]$. Общие формулы для определения количества неизвестных элементов (16) и их расположения (15) остаются в силе и для этих разновидностей. Но из-за строго фиксированной последовательности выбора матрицы $T$ всю необходимую информацию несут структурные индексы $n_{i}, i=1, \ldots, p$.

A. Выбирая кандидаты в строки матрицы $T$ в последовательности $c_{1}^{T}, c_{2}^{T}, \ldots, c_{p}^{T}, c_{1}^{T} A, c_{2}^{T} A, \ldots$, получим

$$
\bar{l}_{i}=p n_{i}+i-\sum_{\substack{j \\ j<i \\ n_{j}<n_{i}}}\left(n_{i}-n_{j}\right)-\sum_{\substack{j \\ j>i \\ n_{j}<n_{i}}}\left(n_{i}-n_{j}-1\right)
$$

и $l_{j k}>\bar{l}_{i}$, если $j<i, \quad k>n_{i}+1$,

$$
\text { или } j>i, k>n_{i} \text {. }
$$

Определим из уравнений (15) и (20) дополнительные нули в матрице $A$ 


$$
a_{i j k}=0, \text { если } \begin{aligned}
& k>n_{i}+1, \quad j<i, \\
& k>n_{i},
\end{aligned}
$$

а из уравнений (7), (16) и (19) число неизвестных параметров А-разновидности канонической формы Луэнбергера

$$
N^{A}=n(m+p)-\sum_{i=1}^{p}\left[\sum_{\substack{j \\ j<i \\ n_{j}<n_{i}}}\left(n_{i}-n_{j}\right)+\sum_{\substack{j \\ j>i \\ n_{j}<n_{i}}}\left(n_{i}-n_{j}-1\right)\right] .
$$

Минимум числа $N^{\text {A }}$ для минимально наблюдаемых систем достигается при $n_{1}=n_{2}=\ldots=n_{p-1}=1, n_{p}=n-p+1$ и равняется

$$
N_{\min }^{\mathrm{A}}=n(m+1)+p(p-1),
$$

а максимум числа $N^{\mathrm{A}}$ достигается при $n_{1}=\ldots=n_{r}=n-r / p+1$, $n_{r+1}=\ldots=n_{p}=n-r / p$, где $r$ есть минимальное число такое, что $n-r$ делится на $p$ без остатка, и равняется

$$
N_{\max }^{\mathrm{A}}=n(m+p) .
$$

Б. Выбирая кандидаты в строки матрицы $T$ в последовательности $c_{1}^{T}, c_{1}^{T} A, \ldots, c_{1}^{T} A^{n_{1}}, c_{2}^{T}, \ldots, c_{2}^{T} A^{n_{2}}, \ldots$, получим каноническую форму Бюси $\left[{ }^{2}\right]$. Тогда

$$
\bar{l}_{i}=\sum_{j=1}^{i} n_{j}+i
$$

и

$$
l_{i k}=\sum_{j=1}^{i} n_{j}+i+k-1 .
$$

Значит, $l_{j k}>\bar{l}_{i}$, если $j>i$, и, согласно (16), $a_{i j k}=0, j>i, k=1, \ldots$ $\ldots, n_{j}$.

Из уравнений (7), (16) и (24) после несложных преобразований получим формулу для определения количества неизвестных параметров в канонической форме Бюси

$$
N^{\mathrm{b}}=n m+\sum_{j=1}^{p}(p-j+1) n_{j} .
$$

Минимум числа $N^{\text {Б }}$ для минимально наблюдаемых систем достигается при $n_{1}=n_{2}=\ldots=1, n_{p}=n-p+1$ и равняется

$$
N_{\min }^{\mathrm{b}}=n(m+1)+p(p-1) / 2 \text {, }
$$

а максимум соответственно при $n_{1}=n-p+1, n_{2}=n_{3}=\ldots=n_{p}=$ $=1$ и равняется

$$
N_{\max }^{\mathrm{b}}=n(m+p)-p(p-1) / 2 .
$$

Сравнение формул (17), (22), (26) и (18), (23), (27) показывает, что для минимально наблюдаемых систем

$$
\begin{aligned}
& N_{\min }=N_{\min }^{\mathrm{b}} \leqslant N_{\min }^{\mathrm{A}}, \\
& N_{\max }^{\mathrm{b}} \leqslant N_{\max }^{\mathrm{A}}=N_{\max } .
\end{aligned}
$$


Числа $N^{\mathrm{A}}$ и $N^{\mathrm{B}}$ в формулах (21) и (25) не подлежат сравнению, так как структурные индексы $n_{i}, i=1, \ldots, p$, имеют различные значения для разных разновидностей канонических форм Луэнбергера. Кроме того, они могут меняться в порядке нумерации выходов.

Покажем, что при наилучшей последовательности выходов каноническая форма Бюси имеет минимальное число неизвестных параметров среди разновидностей канонической формы Луэнбергера.

Пусть

$$
\begin{array}{ll}
\text { ранг } & {\left[c_{i}^{T}, c_{i}^{T} A, \ldots, c_{i}^{T} A^{n}\right]=n_{i}^{*},} \\
\text { ранг } & {\left[c_{i}^{T}, \ldots, c_{i}^{T} A^{n}, c_{j}^{T}, \ldots, c_{j}^{T} A^{n}\right]=n_{i j}^{*} \text { и т. д. }}
\end{array}
$$

и пусть для некоторой разновидности канонической формы Луэнбергера

$$
\bar{l}_{i_{1}}<\bar{l}_{i_{\mathrm{z}}}<\ldots<\bar{l}_{i_{p}}, \quad i_{j} \in\{1, \ldots, p\} .
$$

Тогда из процедуры выбора матрицы $T$ следует, что

$$
\begin{aligned}
\bar{l}_{i_{1}} \geqslant n_{i_{1}}^{*}+1, \\
\bar{l}_{i_{2}} \geqslant n_{i_{1} i_{2}}^{*}+2, \\
\vdots \\
\cdot \\
\cdot \\
\bar{l}_{i_{p}}=n_{i_{1}, \ldots, i_{p}}^{*}+p=n+p .
\end{aligned}
$$

Суммируя обе стороны системы (29), получим

$$
\sum_{j=1}^{p} \bar{l}_{i_{j}}=\sum_{i=1}^{p} \bar{l}_{i} \geqslant n_{i_{1}}^{*}+n_{i_{1} i_{2}}^{*}+\ldots+n+p(p+1) / 2 .
$$

Из формулы (16) и неравенства (30) следует, что для всех разновидностей канонической формы Луэнбергера, сохраняющих соотношения порядка (28), имеет место

$$
N \geqslant n m+n_{i_{1}}^{*}+n_{i_{1} i_{2}}^{*}+\ldots+n_{i_{1}, \ldots, i_{p}}^{*}
$$

Нумеруя выходы так, что $i_{1}=1, i_{2}=2, \ldots, i_{p}=p$, для канонической формы Бюси получим

$$
\begin{aligned}
\bar{l}_{i_{1}} & =\dot{n}_{i_{1}}^{*}+1, \\
\bar{l}_{i_{2}} & =\dot{n}_{i_{1} i_{2}}^{*}+2, \\
& \vdots \\
\cdot & \cdot \\
\bar{l}_{i_{p}} & =n_{i_{1}, \ldots, i_{p}}+p .
\end{aligned}
$$

Значит, при наилучшей нумерации выходов

а с учетом неравенства (31)

$$
N^{\mathrm{b}}=n m+n_{i_{1}}^{*}+n_{i_{1} i_{2}}^{*}+\ldots+n_{i_{1}, \ldots, i_{p}}^{*},
$$

$$
N^{\mathrm{5}} \leqslant N \text {. }
$$

П р и м ер. Пусть $n=4, p=2$ и матрица наблюдаемости (8) имеет вид 


$$
\Im^{T}=\left[\begin{array}{ll:ll:ll:ll}
1 & 0 & 0 & 0 & 1 & 0 & 1 & 0 \\
0 & 1 & 0 & 0 & 0 & 1 & 0 & 1 \\
0 & 0 & 0 & 1 & 0 & 1 & 0 & 2 \\
0 & 0 & 1 & 0 & 1 & 0 & 2 & 0
\end{array}\right]
$$

Выбирая кандидаты в матрицу $T$ в последовательности

$$
l_{11}=1, l_{21}=2, l_{12}=3, l_{22}=4, \bar{l}_{1}=5, \bar{l}_{2}=6,
$$

получим $n_{1}=2, n_{2}=2$. А-разновидность канонической формы Луэнбергера принимает вид

$$
A=\left[\begin{array}{ll:ll}
0 & 1 & 0 & 0 \\
a_{111} & a_{112} & a_{121} & a_{122} \\
\hdashline 0 & 0 & 0 & 1 \\
a_{211} & a_{212} & a_{221} & a_{222}
\end{array}\right], \quad C=\left[\begin{array}{ll:ll}
1 & 0 & 0 & 0 \\
0 & 0 & 1 & 0
\end{array}\right], \quad B=\left[b_{i j}\right]
$$

причем $N^{\mathrm{A}}=4 m+8$. В действительности $a_{111}=a_{112}=a_{221}=a_{222}=1$, $a_{121}=a_{122}=a_{211}=a_{212}=0$.

Выбирая кандидаты в матрицу $T$ в последовательности $l_{11}=1$, $l_{12}=2, \bar{l}_{1}=3, l_{21}=4, l_{22}=5, \bar{l}_{2}=6$, получим $n_{1}=2, n_{2}=2$.

Каноническая форма Бюси имеет вид

$$
A=\left[\begin{array}{ll:ll}
0 & 1 & 0 & 0 \\
a_{111} & a_{112} & 0 & 0 \\
\hdashline 0 & 0 & 0 & 0 \\
a_{211} & a_{212} & a_{221} & a_{212}
\end{array}\right], \quad C=\left[\begin{array}{ll:ll}
1 & 0 & 0 & 0 \\
0 & 0 & 1 & 0
\end{array}\right], \quad B=\left[b_{i j}\right]
$$

причем $N^{\mathrm{5}}=4 m+6$. В действительности $a_{111}=a_{112}=a_{221}=a_{212}=1$, $a_{211}=a_{212}=0$.

\section{Заключение}

Канонические формы описания многомерной динамической системы (1) позволяют сокращать количество неизвестных параметров в матрицах $A, B$ и $C$. Изучению подлежала каноническая форма Луэнбергера с фиксированием элементов матриц $A$ и $C$.

Структура канонической формы фиксируется структурными индексами $n_{i}, i=1, \ldots, p$. Показано, что индексы $l_{i k}$ и $\bar{l}_{i}, k=1, \ldots, n_{i}$, позволяют находить расположение еще $\sum_{i=1}^{p}\left(n+i-\bar{l}_{i}\right)$ нулевых элементов.

Следует отметить, что дополнительных вычислений для определения индексов $l_{i k}$ и $\bar{l}_{i}$ не требуется, т. к. они выясняются в процессе отыскания структурных индексов $n_{i}$. Необходима лишь дополнительная память для хранения $n$ чисел.

У конкретных разновидностей канонической формы Луэнбергера достаточную информацию несут структурные индексы. Сравнение разновидностей показало, что выбор подходящей нумерации выходов приводит систему к канонической форме Бюси, имеющей наименьшее число неизвестных параметров. 


\section{Л ИТ Е РАТ У Р А}

1. Tether A. J., Larson R. E., Proc. of 4th Hawaii Intern. Conf. on System Sciences, 1971, p. 93.

2. Bucy R. S., A ckerm a n n J., Regelungstechnik, 18, N 10, 451 (1970).

3. Luenberger D. E., IEEE Trans. Automatic Control, 12, No. 3, 290 (1967).

4. Gupta R. D., Fa irman F. W., IEEE Trans. Automatic Control, 19, No. 4, 440 (1974).

Ннститут кибернетики Академии наук Эстонской ССР
Поступила в редакцию

8/VI 1976

\section{O. NURGES}

\section{MITMEMÓTTMELISE DUNAAMILISE SUSTEEMI PARAMEETRITE ARVUST}

Vaadeldakse dünaamilise süsteemi Luenbergeri kanoonilist kuju. Struktuuriindeksid $n_{i}$, kus $i=1, \ldots, p$, võimaldavad fikseerida $n^{2}$ parameetri väärtuse ja koha. Indeksid $l_{i k}$ ja $\bar{l}_{i}$, kus $k=1, \ldots, n_{i}$, mis seiguvad struktuuriindeksite määramisel, võimaldavad peale selle fikseerida

$$
\sum_{i=1}^{p}\left(n+i-\bar{l}_{i}\right)
$$

nulli Luenbergeri kanoonilises kujus. Näidatakse, et väljundite sobiva nummerdamise puhul sisaldab Bucy kanooniline kuju minimaalse arvu tundmatuid parameetreid Luenbergeri kanooniliste kujude klassis.

\section{NURGES}

\section{ON THE NUMBER OF PARAMETERS OF MULTIDIMENSIONAL DYNAMICAL SYSTEM}

Luenberger's canonical form of the $n$-th order linear system with $m$ inputs and $p$ outputs is considered. It is well known that $n^{2}$ parameters of the canonical form are fixed by structural indices $n_{i}, i=1, \ldots, \mathrm{p}$. As byproducts of the procedure for determining the structural indices, the indices $l_{i k}$ and $\bar{l}_{i}, \mathrm{k}=1, \ldots, n_{i}$, are determined and used for reducing the number of unknown parameters in Luenberger's canonical form. There is a great number of modifications of Luenberger's canonical form. It is shown that one of them, namely Bucy's canonical form, has the minimal number of unknown parameters if the ordening of outputs is appropriately chosen. 